\title{
An Architecture for Interactive Target-Oriented E/M-Learning Systems
}

\author{
doi:10.3991/ijet.v4i4.782 \\ C. De Castro, G. Leonardi and P. Toppan \\ IEIIT-CNR, Italian National Research Council, Bologna, Italy
}

\begin{abstract}
This paper proposes an architecture to develop an e/m-Learning system with the following features: (1) learning paths that can be tailored to the user's aims, which can be dynamically modified during the learning process to meet user needs; (2) in the context of m-Learning functionalities, contents that can be scaled and adapted to the user's device and connection technology. As far as learning paths are concerned, learners are allowed to specify their initial target and, if they decide to study some issues at depth, they can gain authorization to redefine learning targets over time. This process is guided by a "Path Decision Module" based on Petri Nets, which checks the users' learning level and consequently decides whether further learning materials can be faced or not. As for access to the system is concerned, the following scenarios are considered: UMTS (e.g.: for PDAs), DSL (e.g.: at home), WiFi (e.g.: within study lounges and libraries), wired/fiber (e.g.: within laboratories). In order to satisfy such heterogeneous requirements, contents are dynamically adapted to available technologies by means of on the fly data format conversions. The whole system relies on a hybrid LDAP-SQL database which uses directory services and relational databases. LDAP is used to store learning and assessment materials, whereas dynamic paths are stored in the SQL system. Analytical calculations are also presented which show the access time in each scenario and, consequently, if a certain activity can efficiently be carried out or not.
\end{abstract}

Index Terms - e/m-Learning, personal targets, data format scaling, Petri Nets, LDAP-SQL.

\section{INTRODUCTION}

Advanced e-Learning systems must take many factors into consideration, such as functions offered the user, organization of contents and service fruition modalities [1]-[8].

As far as functions are concerned, it is becoming more and more important to involve the user actively and to adapt the study process to specific needs. Not only does such a requirement imply a personalized and careful definition of learning paths, but also an efficient choice of assessment phases [9]-[13].

If subjects and contents are taken into consideration, whose management is the core of any e-learning system, among the many properties they should meet include clarity, completeness and their possible extension and integration with other learning sources. Of course, such features must be taken into great consideration when choosing the kind of database, for instance a relational system rather than an object-oriented or a multimedia one.
As for accessing the e-Learning system, modalities should be as diversified as possible on the basis of student aims, ties, and timetables, as well as location and technologies at the user's disposal. All such factors are decisive in order to reach a good compromise between quality of learning and studying conditions [14]-[19].

In more detail, the following scenarios are taken into account:

1. UMTS: used often when accessing the system through PDAs, for instance if some exercises must be realized;

2. DSL: typically used when users access the system from a home setting;

3. WiFi: quite common within a campus, in study lounges, and libraries;

4. wired/fiber: generally used in the case of universities (for instance, in laboratories).

In short, the entire architecture has been designed in order to meet the following requirements:

\section{Contents:}

i. first, according to user interests and purposes, users can define a personalized target or learning level;

ii. moreover, in case individual users discover further interesting issues or more in-depth knowledge, the eLearning path can change accordingly over time.

\section{Access modalities:}

iii.in addition, learners are allowed to use different devices and different connection technologies so the data is scaled and adapted for different situations.

Taking all the above factors into consideration, the proposed architecture consists of the following overlying layers:
a) access to the system;
b) testing module and learning path decision;
c) data format adaptation module;
d) database and organization of contents

Personalized targets and individual interests can be achieved through modules (a), (b) and (d); the use of heterogeneous devices is made possible by modules (c) and (d). In more detail, the learning process is controlled by a Petri Net, which checks whether prerequisites have been met or not. As far as data scaling is concerned, it is fulfilled by on the fly data format conversions. As for information, the whole system is based on a hybrid LDAP/SQL database, storing learning and assessment material, as well as customized studying paths. Such operations are meant 
to be tailored to different situations and last the whole lifespan of the learning process itself.

The constant interaction among layers allows the system to adapt learning paths dynamically, with respect to both subjects and studying conditions. In particular, specific tests are constantly defined to keep improvements under constant check and the user has a certain degree of freedom in choosing access modalities.

This approach could be of help in many fields, including Computer-Aided Assessment, which, ranging from automated multiple-choice tests to more sophisticated systems, is becoming increasingly useful. With some such systems, feedback can be defined on the basis of both mistakes and actual achievements and can also be adapted to the natural mobility of many workers.

More generally, these early considerations about access modalities can be of help in the context of heterogeneous learning/teaching methodologies.

The paper is organized as follows: first, the proposed architecture is described from the user viewpoint and dynamic path decision (Section II). Afterwards, the whole architecture is presented and its components detailed (Section III). In particular, the integrated e/m-learning schema is discussed with respect to data format scaling, testing phases and the underlying LDAP/SQL database technologies. Section IV presents analytical calculations of response time on the basis of access connections, showing which parts of the e-learning process can be considered feasible in each considered scenario.

\section{MAIN ARCHITECTURE}

The proposed architecture is firstly described with respect to $\mathrm{I} / \mathrm{O}$, then to the data flow which takes place over time among the four layers outlined in Section 1. The static behavior of the system is depicted in Fig. 1, whereas the dynamic process is represented in Fig. 2.

Each layer will be expanded afterwards to discuss its components.

A first, rough description of the whole architecture is shown in Fig. 1, where two main components are considered: the "User-System Communication Interface" (A) and the "Learning System Kernel" (B).

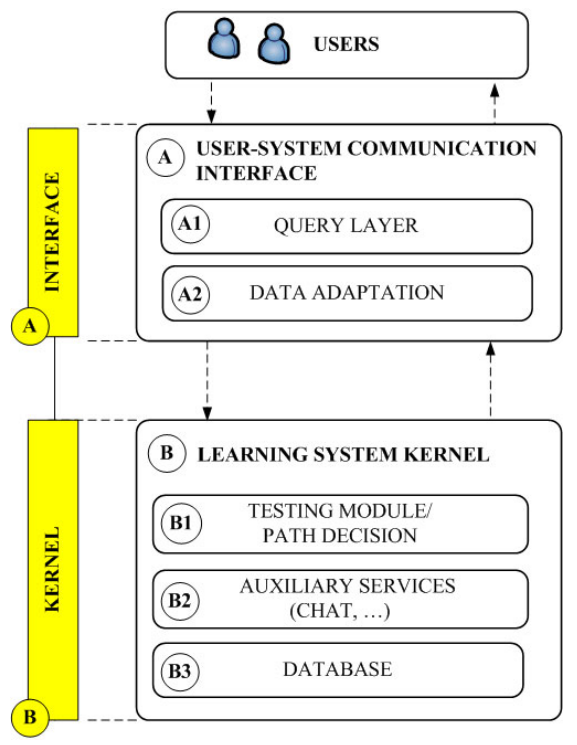

Figure 1. Main architecture
The main aim of the first component is to facilitate data exchange between the user and the system. In this context, the word user refers to a student who queries the system during a learning process or asks for the availability of further services. Such functionality is represented by the "Query Layer" module (A1).

The second role of the Communication Interface is to optimize the data exchange quantity and format between the user and the system to fit different connections and devices. This process is represented by the "Data Adaptation" module (A2).

As far as the Learning System Kernel is concerned, it is composed of: the "Testing Module/Path Decision" block (B1). Further services are placed at the student's disposal, including a chat application for contacting teachers and other similar applications (B2). The third component (B3) is an integrated database that stores test and study files, as well as personalized learning plans and bookings of services

The overall process is the following: the user chooses a target, which is forwarded to the kernel. On the basis of the specified target, said module decides the initial test the user must undergo and successively suggests appropriate learning steps.

Both the test and study files are consequently retrieved from the database and - as long as they are compatible with the e-Learning plan - adapted to the user's device and connection technology.

The dynamic definition of learning paths is a process that continues by means of further assessment phases and suggested steps. In case users decides to modify their target, they notify the system of the new information.

In order to better explain how such process evolves over time (Fig. 2), let us define:

- $\mathrm{T}_{\mathrm{i}}$ the $\mathrm{i}$-th target defined by the user, $\mathrm{T}_{0}$ being the initial target;

- $\mathrm{L}_{\mathrm{ij}}$ the $\mathrm{j}$-th learning level tested during the learning phase whose target is $T_{i}$;

- $\mathrm{S}_{\mathrm{ij}}$ the $\mathrm{j}$-th studying step suggested during the learning phase whose target is $T_{i}$

When $T_{i}(i=0,1 . . n)$ and all the information about the user's technology is forwarded from the external layer to the Testing Module. Said block then accesses the database, retrieves an assessment test appropriate to $T_{i}$ and the whole situation and proposes what to do to the user.

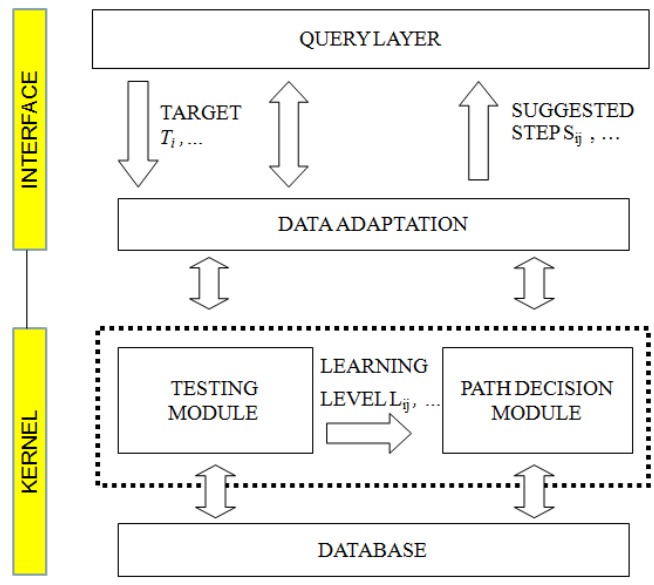

Figure 2. Main architecture: I/O data flow over time 
On the basis of the test results, a learning level $\mathrm{L}_{\mathrm{ij}}$ is decided and the Path Decision Module is notified. Said module then accesses the database and retrieves a learning step $\mathrm{S}_{\mathrm{ij}}$ that is appropriate to $\mathrm{T}_{\mathrm{i}}, \mathrm{L}_{\mathrm{ij}}$ and adapted to both the user's devices and access technologies.

$\mathrm{S}_{\mathrm{ij}}$ is finally proposed to the user and the process continues with further testing phases and definition of further learning levels and steps $(j=1, . . \mathrm{m}$ for each fixed $\mathrm{i})$.

\section{MAIN ARCHITECTURE EXPANDED}

In this section, the architecture in Fig. 1 is refined and its components discussed separately. The proposed approach can be described by means of the 4-layered architecture in Fig. 3, which is increasingly enriched from the left to the right.

The vertical axis on the left reflects the classification in Fig. 1. On the right, all the components are expanded and represented with respect to their interaction and to the data flow which takes place among them.

The main purpose and features of each block and its submodules are described in the following.

\section{A. User-System Communication Interface}

The User-System Communication Interface must carry out the following tasks:

1. receive the user's requests (initial target and successive ones) and forward them to the Testing Module. All such requests are meant to be events that must be relayed to the system;

2. receive the suggested assessment tests from the Testing Module and return the results;

3. receive the suggested learning steps from the Path Decision Module and allow the user to access the study material;

4. manage access to auxiliary services;

5. transform all such requests and answers based on the user's devices (mobile, PDA, laptop, etc.). In other words, make the user and the system communicate on the basis of the user's device ("Device Interpretation");

6. adapt learning data to the user's device and access technology to meet the learner's requirements and optimise the overall process ("On the Fly Data Conversion").

Note that task 5 concerns the management of queries and data with respect to the user's device and that it is accomplished by the Query Layer. Task 6 concerns data optimization on the basis of network access technology and is fulfilled by the Data Adaptation module.

In more detail, the problem of converting data on the basis of available devices has not been mentioned yet. However, when talking of a data format, two different even if not independent - operations must be distinguished: first, data transformation due to the type of device (Data Interpretation on the basis of device); second, data format optimization due to the type of network connection (Data Adaptation).

As for data interpretation, the system must communicate on the basis of the user's device. Consequently, the user's data must be converted into a format that both the front-end to the system and the database can understand. This process lasts the entire lifespan of the learning pro-

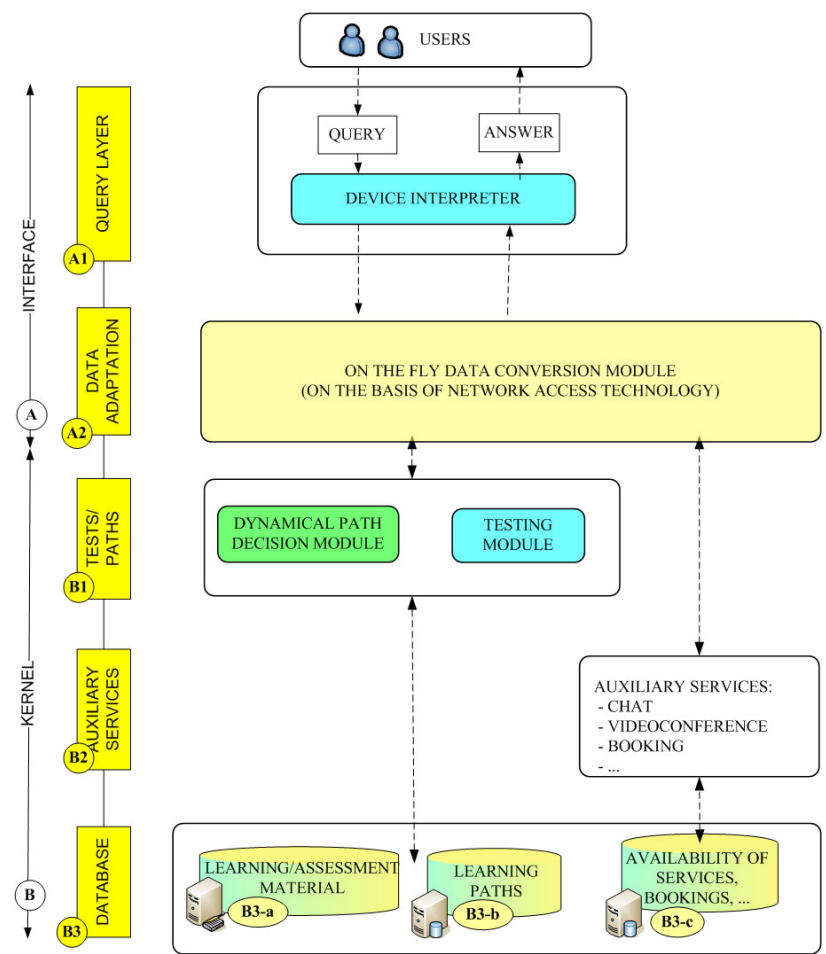

Figure 3. Main components of each layer

cess and can easily be done by means of XML conversions. As a matter of fact, this is a straightforward, general and effective way of exchanging data between heterogeneous environments.

As far as the Data Adaptation module is concerned, in order to optimize response time in each scenario, and especially in order to support m-Learning, the following guidelines must be taken into account: first, it is essential to reach the best trade-off among the user's actual needs with respect to the quality and quantity of data, as well as response time; second, information must be adapted to available technologies (PDAs versus laptops, etc.).

Developing such a system involves at least two aspects: first, information must be represented at different levels using different formats; second, an access methodology must be designed for filtering data on the basis of the above criteria. This is accomplished by the On the Fly Data Conversion Module, which retrieves data in its original format and scales it so as to adapt it to the kind of network access technology and device.

Some considerations about these issues are the core of Section IV.

\section{B. Path Decision and Assessment Module}

As discussed in the description of the whole architecture, the main role of this layer is to be aware of the user's aims, check his/her learning levels, and consequently define a tailored study path.

The main idea is the strict interaction between the Path Decision/Assessment Layer and the database. As a matter of fact, the database stores both assessment and study material which are selected from the database on the basis of the user's goals and actual achievements.

The database schema will be discussed in Section C, however, in order to describe the process, it is briefly summed up below. 
Its contents can be represented by means of a network of issues, levels and prerequisites. Consider issues $\mathrm{I}_{1}$ and $\mathrm{I}_{\mathrm{m}}$ and suppose they are related (such as derivatives and integrals) and meant to be worked with at a given level (such as a course of Mathematics at a high school).

For the sake of simplicity, suppose $\mathrm{I}_{1}$ and $\mathrm{I}_{\mathrm{m}}$ can be considered steps of the learning process.

Suppose that facing step $\mathrm{I}_{\mathrm{m}}$ after step $\mathrm{I}_{1}$ requires prerequisites $\mathrm{p}_{1}, \mathrm{p}_{2}, . . \mathrm{p}_{\mathrm{k}}$.

This kind of algorithm can be represented by means of a Petri Net [20]-[23], which acts as a traffic light between a learning step and the successive one.

In this approach, the Petri Net's places are testing phases (prerequisites), and its transitions are studying materials. A transition is enabled if all the required prerequisites have been fulfilled.

In other words, the architecture in Fig. 2 hides a cycle that guides the assessment phase and the possibility or not to proceed to further steps.

Since the system is adaptive, contents are put at the user's disposal on the basis of his/her device and access technology.

\section{Information System Layer: why LDAP/SQL}

As far as the underlying e-learning information system is concerned, its architecture has been designed keeping in mind that two kinds of information are involved:

i. e-learning and assessment material, which is not meant to be frequently updated (named static data);

ii. personalised learning paths and assessment results, which are time-varying (named dynamic data);

It must also be noticed and considered that e-learning information can be represented by means of a hierarchy of subclasses; for instance, from a subject to its specific issues.

These requirements suggest the use of a hybrid database structure for data storage: an LDAP directory service [24][25] for static data and a relational DBMS for dynamic information.

LDAP provides both a model and an implementation tool that is particularly suitable for web-based e-learning applications, both from the data representation viewpoint and for an efficient web-based access.

As a matter of fact, it is scalable, extendable and optimised for reading operations, so it is particularly suitable for static data. It also supports standards and interfaces of many multimedia broadband applications and integrated access to e-learning services.

Another important feature is that LDAP represents information by means of a hierarchy of classes using very flexible schemata. In the considered environment, this implies at least three advantages.

First, the knowledge that a person acquires on a specific subject can be organized in an LDAP tree as follows: the $\mathrm{n}^{\text {th }}$-level class describes the subject in general; the $\mathrm{n}^{\text {th+1 }}$ level classes represent related subjects, issues and related issues, documentation and assessment material, prerequisites, learning paths, and assessment tests.

Learning and testing material, as well as paths and tests, are divided into as many subclasses as the number of tar- get levels provided for. In this way the middle layer can access the correct material.

As for the second advantage, LDAP was built for the integration of distributed environments, so it also suits the distributed location of documentation very well. As a matter of fact, for applications such as international remote education, e-learning information is distributed by nature.

The third advantage concerns schema management. The schema of the e-learning database is likely to be modified or augmented during its life cycle as, for instance, due to the addition of new kind of media or information described by different properties. A relational system, in traditional settings, does not allow efficient schema revision. Such operations involve high costs in terms of redesigning existent schemata, reloading data and verifying that original constraints and relationships on data are preserved. LDAP, on the contrary, offers high flexibility in modifying data structures.

As far as the dynamic part of the database is concerned, it mainly concerns the time-varying personalised learning paths. In more detail, the dynamic database stores information about the users and their individual learning phases, such as targets, suggested steps and actual achievements.

Other information involves auxiliary services and their booking.

In this case, an SQL database is more suitable. As a matter of fact, such models are optimized for reading/writing operations and time-varying data.

The connection between the LDAP and the SQL databases are LDAP object identifiers which, as identifiers of subjects, issues, etc., are used as key information that defines dynamical paths and assesses tests results. They are also used in the joint navigation of LDAP and SQL data.

\section{ANALYTICAL CALCULATIONS}

Some considerations will now be made on the basis of: (a) scenario, meaning connection speed; (b) some learning contents and their dimensions; (c) minimal QoS required for a learning activity to be carried out efficiently.

As for connection speed, four situations are considered (Table I). In this context, download speed should be more properly called goodput, i.e. throughput at the application level.

TABLE I.

SCENARIOS AND CONNECTION SPEEDS

\begin{tabular}{|l|l|l|}
\hline \multicolumn{1}{|c|}{ Scenario } & \multicolumn{1}{|c|}{ Technology } & \multicolumn{1}{c|}{$\begin{array}{c}\text { Download } \\
\text { speed (peak) }\end{array}$} \\
\hline with PDAs & UMTS & $1 \mathrm{Mbps}$ \\
\hline $\begin{array}{l}\text { study lounges, } \\
\text { libraries (lap- } \\
\text { tops) }\end{array}$ & $\mathrm{WiFi}$ & $5 \mathrm{Mbps}$ \\
\hline $\begin{array}{l}\text { at home (lap- } \\
\text { tops, desktops) }\end{array}$ & DSL & $7 \mathrm{Mbps}$ \\
\hline $\begin{array}{l}\text { laboratories } \\
\text { (laptops, desk- } \\
\text { tops) }\end{array}$ & wired/fiber & $10 \mathrm{Mbps}$ \\
\hline
\end{tabular}

The contents considered in this model are summed up in Table II: exercises, lecture notes and seminars, all of which are meant to be downloaded and used off line. 
TABLE II.

DiMENSIONS IN KB OF LEARNING DATA

\begin{tabular}{|c|c|}
\hline Data & Dim(MB) \\
\hline exercise & 1 \\
\hline lecture notes & 5 \\
\hline seminar & 10 \\
\hline
\end{tabular}

QoS thresholds are indicated in Table III, where three online activities are considered: following a lecture through videoconference, taking an exam on the Internet, and brainstorming with fellow students. The threshold indicates the minimal connection speed required for the acceptable fruition of the online learning material.

TABLE III.

QOS THRESHOLD

\begin{tabular}{|c|c|}
\hline Activity & QoS threshold (Mbps) \\
\hline lecture & 1 \\
\hline examination & 6 \\
\hline brainstorming & 8 \\
\hline
\end{tabular}

The above parameters are approximate. Nevertheless these considerations and the following calculations are meant to guide the user in choosing the appropriate learning conditions. The considerations above are formalized in Fig. 4.5, which shows the download time in the four scenarios and the activities that are feasible or not.

As for Fig. 4, The $\mathrm{x}$-axis represents learning data and scenarios, and the $y$-axis represents download time. Within each scenario, the blue area (the first one in the row) represents exercises; the purple area (second in the row) represents lecture notes; the yellow area (third in the row) represents seminars.

Comparing the four scenarios of UMTS, WiFi, DSL and wired/fiber, an exercise can be downloaded in 8 (UMTS, 1Mbps), 1.6 (WiFi, 5 Mbps), 1.1 (DSL, 7 Mbps) and 0.8 (wired/fiber, $10 \mathrm{Mbps}$ ) seconds respectively. Lecture notes require $40,8,5.7$ and 4 seconds. A seminar requires $80,16,11.4$ and 8 seconds.

As far as Fig. 5 is concerned, following a lecture is hardly feasible with an UMTS technology, but it is feasible using the others.

Taking an exam can be done efficiently with a good DSL and fiber connection, whereas UMTS and WiFi are not acceptable.

Brainstorming activities are only be effected with a wired/fiber connection.

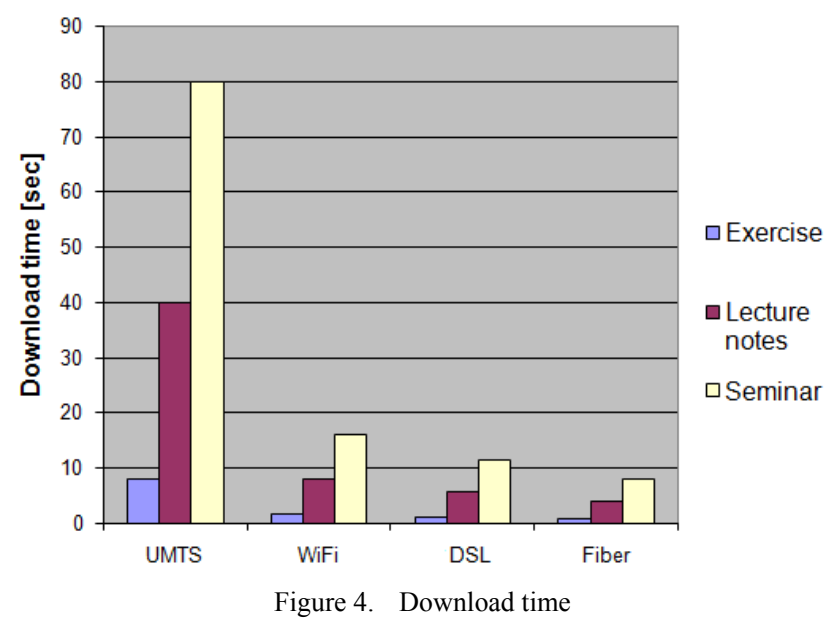

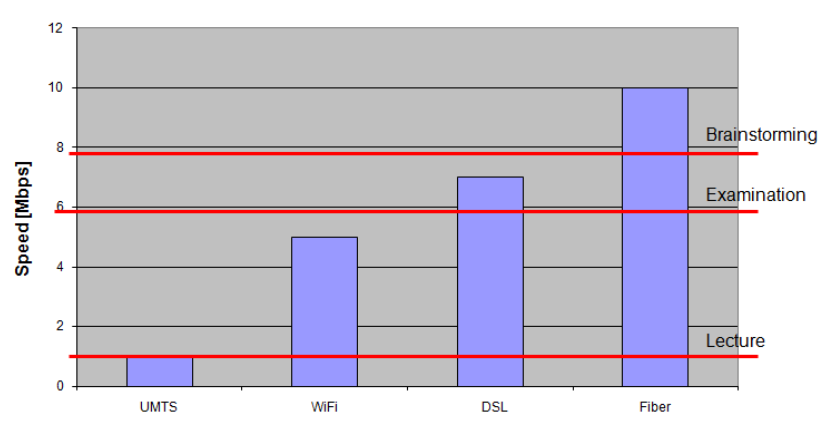

Figure 5. Feasibility

\section{CONCLUSION}

In this paper, a three-layered architecture was discussed for an e-learning system where users are allowed to specify their goals and redefine them during the learning process. The proposed architecture is based on an LDAP-SQL information system which interacts with an assessment and path decision module. Said block suggests appropriate steps, retrieved from the database, based on the user's actual achievements.

Computations were also presented to assist the student select the information that can be effectively downloaded, as well as activities that are feasible or not in the considered scenarios.

Future work will be devoted to the application of the proposed architecture to the TELEDOC2 project, whose objective is to define specialized learning paths for Information and Communications Engineering $\mathrm{PhD}$ students and to make them accessible through multimedia services.

\section{REFERENCES}

[1] J. Scrivener, "Learning Teaching", Macmillan Education, 1994.

[2] C. Handy, "Beyond Certainty", Harvard Business School Press, 1995.

[3] D. Laurillard, "Rethinking university teaching: a conversational framework for the effective us of learning technologies", London, Routledge-Farmer, 2002, $2^{\text {nd }}$ ed.

[4] D. Kay, "E-learning: Drivers, Developments, and Decisions", Multimedia Information and Technology, vol. 29, no. 1, pp. 26-29, 2003.

[5] M. Eppler, "Knowledge Visualisation: Towards a new Discipline and its Fields of applications", \#2/2004 ICA Working Paper, University of Lugano, (USI), Switzerland, City, 2004.

[6] K. Moore, L. Aspen, "Coping, adapting, evolving: the student experience of e-learning". Library and Information Update, vol. 3, no. 4, pp. 22-24, 2005.

[7] A. Tombros, I. Ruthven I., J. M. Jose, "How users assess web pages for information seeking", Journal of the American Society for Information Science, ACM 2005.

[8] S. Bocconi, S. Dini, L. Ferlino, et al, "Accessibility of Educational Multimedia: in search of specific standards", International Journal of Emerging Technologies in Learning (iJET), Vol. 1, No. 3, 2006, http://online-journals.org/i-jet/.

[9] S. P. Christodoulou, D. M. Garyfallidou, G. S. Ioannidis et al, "Interactive education based on haptic technologies and educational testing of an innovative system", International Journal of Emerging Technologies in Learning (iJET), Vol. 3 No. 2, 2008, http://online-journals.org/i-jet/.

[10] N. Henze, "Personalized E-Learning in the Semantic Web", International Journal of Emerging Technologies in Learning (iJET), Vol. 1, No. 1, 2006, http://online-journals.org/i-jet/.

[11] E. Kongar, A.A. Zaghleh, T. Sobh, "Online Customization and Enrollment Application Network (OCEAN)", International Journal of Emerging Technologies in Learning (iJET), Vol. 2, No. 3, 2007, http://online-journals.org/i-jet/. 
[12] C. De Castro, P. Toppan, "Dynamical Target-Oriented e-Learning Networks", Proc. of International Conference on Technology, Education and Development (INTED 2008), Valencia, Spain, March, 3rd-5th 2008.

[13] C. De Castro, P. Toppan, "An Architecture for Interactive TargetOriented e-Learning Systems", Proc. of 50th International Symposium ELMAR-2008, Zadar, Croatia, 10-12 September 2008.

[14] S. Heier, D. Heinrichs, A. Kemper, "Performance evaluation of Internet applications over the UMTS radio interface", Proc. of Vehicular Technology Conference, IEEE VTC 2002, 4,1834-1838, 2002.

[15] G. Bronson, K. Pahlavan, H. Rotithor, "Performance evaluation of wireless LANs in the indoor environment", Proc. of 18th Conference on Local Computer Networks, 452 - 460, 1993.

[16] M.C. Yuang, , S.J. Hsu, "LAN protocol modelling and performance evaluation", Communications, ICC 94, SUPERCOMM/ICC '94, IEEE International Conference on Serving Humanity Through Communications, 2, 685 - 689, 1994.

[17] N. Caouras, M. Freda, F. Monfet, V.S. Aldea, O. Naeem, L. Tho, B. Champagne, "Performance evaluation platform for xDSL deployment in a complex multi-segment environment", Proc. of Canadian Conference on Electrical and Computer Engineering, 2003. IEEE CCECE 2003, 1, 61 - 64, 2003.

[18] C. Donzelli, C. Fontana, A. Ravaioli, P. Toppan, M. Patella, C. De Castro, "An LDAP/SQL-based Architecture for Broadband Services", Proc. of IASTED Int. Conference on Communication Systems and Applications (CSA 2006), Banff, Canada, pp. 96-101, July 3rd-5th 2006.

[19] C. De Castro, P. Toppan, "Adaptive Release of Braoadband Services for the Management of Medium-Priority Applications", Proc. of the 18th Annual IEEE International Symposium on Per- sonal, Indoor and Mobile Radio Communications (PIMRC'07), Athens, Greece, September 2007.

[20] C. S. Chen, Y L. Ke, J. S.Wu, "Coloured Petri nets approach for solving distribution system contingency by considering customer load patterns", IEE Proc.-Gener Transm. Distrib. Vol. 148, No. 5, September 2001 (doi:10.1049/ip-gtd:20010445)

[21] D. Li, Y. Cui, K. Xu, et al., "Improvement of Multicast Routing Protocol Using Petri Nets", Slezak et al (Eds.): RSFDGrC 2005, LNAI 3642, pp. 634-643, 2005, Springer-Verlag Berlin Heidelberg 2005.

[22] E. Pacuit, R. Parikh, "The Logic of Communication Graphs", Declarative Agent Languages and Technologies (DALT 2004), Lecture Notes in Computer Science, 2004

[23] Hosobe H., "A high-dimensional approach to interactive graph visualization", SAC 2004.

[24] W. Yeong, T. Howes, S. Kille, "Lightweight Directory Access Protocol" IETF RFC 1777, http://www.ietf.org/rfc/rfc1777.txt

[25] T. Howes, M. Smith, G. Good, Understanding and Deploying LDAP Directory Services, Addison Wesley 2003, $2^{\wedge}$ ed.

\section{AUTHORS}

Cristina De Castro, Giacomo Leonardi and Paolo Toppan are with IEIIT-CNR, Italian National Research Council, Dept of Computer Science and Telecommunications, V.le del Risorgimento 3, Bologna, Italy 40136 (email: \{cdecastro,gleonardi,ptoppan\}@bo.ieiit.cnr.it).

Submitted 23 December 2009. Published as resubmitted by the authors on 18 November 2009. 